\title{
KIf4 Alleviates Lipopolysaccharide-Induced Inflammation by Inducing Expression of MCP-1 Induced Protein 1 to Deubiquitinate TRAF6
}

\author{
Zhenzhen Lia Yanhui Jia ${ }^{a} \quad$ Shichao Han ${ }^{a} \quad$ Xingqin Wang ${ }^{b} \quad$ Fu Han ${ }^{a} \quad J u l e i$ Zhang ${ }^{a}$ \\ Wei Zhang ${ }^{\mathrm{a}}$ Hao Guan ${ }^{\mathrm{a}}$ Dahai Hu
}

aDepartment of Burns and Cutaneous Surgery, Xijing Hospital, Fourth Military Medical University, Xi'an, Shaanxi, bDepartment of Neurosurgery, 81th Hospital of PLA, Nanjing, Jiangsu, China

\section{Key Words}

KIf4 • MCPIP1 • Lipopolysaccharide • NF-kB • Deubiquitination

\begin{abstract}
Background/Aims: Inflammation is an essential component of innate immunity against pathogens, but is tightly regulated, such as by Krüppel-like factor 4 (Klf4), to prevent injury. KIf4 also regulates macrophage polarization, although the mechanisms underlying both functions are poorly understood. The aim of this study was to investigate whether and how Klf4 prevents unregulated inflammation. Methods: The bone marrow-derived macrophages and RAW264.7 cell line were used. Quantitative real-time PCR was used to determine inflammatory cytokines (IL-1 $\beta$, TNF- $\alpha$, IL- 6 and MCP-1), Klf4 and MCPIP1 transcript levels. Extraction of nuclear and cytoplasmic proteins and Immunoblotting were used to determine KIf4, MCPIP1, relative kinases from NF-KB pathway and K63-linked polyubiquitins expression in nucleus and cytoplasm separately. Dual luciferase reporter assay was used to analyze whether Klf4 mediate MCPIP1 transcription. Immunoprecipitation was used to determine the protein interaction among KIf4, MCPIP1, TRAF6 and K63-linked polyubiquitins. Secretion of IL-1 $\beta$ and TNF- $\alpha$ into sera in mice was measured by Enzyme-linked immunosorbent assay. Results: We found that exposure to lipopolysaccharides suppresses KIf4 expression, even as it induces release of proinflammatory cytokines. Strikingly, KIf4 overexpression significantly lowered cytokine secretion and NF-KB signaling in the cytoplasm following exposure to lipopolysaccharide, even though KIf4 was exclusively nuclear. The cytoplasmic effects are likely mediated by MCP-1 induced protein 1 (MCPIP1), a deubiquitinase and a key modulator of inflammation that accumulates both in the nucleus and cytoplasm in response to Klf4. Indeed, binding between MCPIP1 and K63 polyubiquitins is attenuated in macrophages overexpressing Klf4, suggesting that MCPIP1 is an intermediator induced by Klf4 in the nucleus to remove K63 polyubiquitins from TRAF6 in the cytoplasm, and thereby impede NF-KB and inflammatory signaling. Importantly, Klf4

Zhenzhen Li, Yanhui Jia and Shichao Han contributed equally to this work.

Dahai Hu

and Hao Guan

Dept. of Burns and Cutaneous Surgery, Xijing Hospital, Fourth Military Medical

University, Xi'an, Shaanxi, (China)

E-Mail dahaihu_burns@163.com / guanhao@hotmail.com
\end{abstract}

KARGER 
overexpression in mice alleviated sepsis symptoms following exposure to lipopolysaccharides. Conclusion: The data highlight KIf4 as an essential MCPIP1-dependent modulator of innate immunity that protects against excessive and self-destructive inflammation.

(C) 2018 The Author(s)

Published by S. Karger AG, Basel

\section{Introduction}

Inflammation plays an important role in the innate immune response to tissue injury or pathogens [1,2], and is induced upon exposure to pathogen- or damage-associated molecular patterns $[3,4]$. Indeed, innate immune cells such as macrophages induce inflammation and produce inflammatory cytokines, including IL-1 $\beta$, TNF, MCP-1, and IL-6, as a first line of defense against viruses or bacteria $[5,6]$. However, unregulated inflammation may also lead to tissue injury, septic shock, autoimmune disease, and malignancy [7]. Therefore, the inflammatory response is precisely controlled at multiple levels to be effective against microbes but also to avoid self-injury.

Lipopolysaccharide, one of the most studied pathogen-associated molecular patterns, is found in the outer membrane of Gram-negative bacteria, and elicits a strong immune response in animals. Generally, lipopolysaccharides engage Toll-like receptor 4 (TLR4), and trigger multiple signaling cascades, ultimately causing a gamut of cell or tissue damage [810]. In one such cascade, binding of lipopolysaccharides to TLR4 triggers auto-ubiquitination of TNF receptor-associated factor 6 (TRAF6) with K63-linked ubiquitin chains $[2,11,12]$. Downstream of TRAF6, TAK1 and the adaptor proteins TAB2 and TAB3 activate the IKK complex, which in turn phosphorylates and hence targets $\mathrm{I} \mathrm{B}$ for proteasomal degradation. This event frees NF- $\mathrm{BB}$ (p50/p65), a transcription factor involved in both innate and adaptive immunity, to translocate to the nucleus, initiate transcription, and trigger release of inflammatory cytokines [13]. Therefore, regulation of ubiquitination of effectors in NF- $\kappa B$ signaling plays a critical role in inflammation.

Ubiquitin is a 76-amino acid polypeptide present in all eukaryotic cells [14-16]. The best characterized forms are K63 polyubiquitins, which mainly control protein-protein interactions [16-19]. Accumulating evidence show that the ubiquitin system, especially deubiquitinases, is essential in immunity [20-22]. For example, TRAF proteins are regulated by MCP-1 induced protein 1 (MCPIP1) [23, 24], a deubiquitinase and zinc-finger protein [2, $25,26]$ that accumulates rapidly upon stimulation with pro-inflammatory agents such as lipopolysaccharides, IL-1, TNF, and MCP-1 [8, 26, 27]. Importantly, MCPIP1 was implicated in both physiological and pathological inflammation [2, 12, 28-31].. Nevertheless, the specific mechanisms linking MCPIP1 to lipopolysaccharide-induced inflammation remain to be identified.

Interestingly, the MCPIP1 promoter contains multiple response elements to Krüppellike factor 4 (Klf4). Accordingly, IL-4 induces MCPIP1 expression via Klf4 [32, 33]. However, the specific regulation between Klf4 and MCPIP1 in the inflammatory response in macrophage is still unclear. Klf4 is well-known not only as a transcription factor essential for pluripotency [34], but also as a regulator of physiological and pathological processes $[35,36]$. In particular, it plays importment roles in macrophage polarization and innate immunology regulation. Notably, Klf4 was demonstrated to promote M2 polarization and inhibit coactivators required to activate NF- $\kappa$ B. Moreover, Klf4-deficient macrophages show increased pro-inflammatory gene expression, enhanced bactericidal activity, and altered metabolism [33]. However, the mechanisms underlying this strict regulation are unclear, further research is needed to address whether and how Klf4 regulates NF- $\mathrm{BB}$ signaling to control the inflammatory response in macrophages.

We now report that Klf4 alleviates the inflammatory reaction to lipopolysaccharide in vitro and in vivo by reducing the release of pro-inflammatory cytokines. We also found that Klf4 accumulates in the nucleus and indirectly inhibits NF- $\kappa B$ signaling by inducing MCPIP1 and thereby elicit deubiquitination of TRAF6 in the cytoplasm. Collectively, our data highlight Klf4 as a promising regulator of inflammation and target of future research. 


\section{Cellular Physiology Cell Physiol Biochem 2018;47:2278-2290

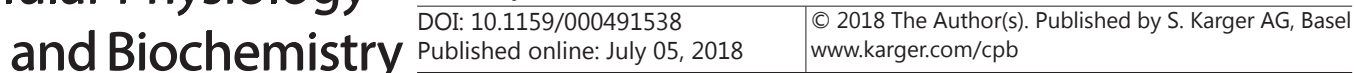

Li et al.: Klf4 Alleviates Inflammation via Modulation of MCPIP1

\section{Materials and Methods}

\section{Animal experiments}

Wild-type male C57BL/C6 mice 6-8 weeks old were purchased from the Experimental Animal Center at Fourth Military Medical University, and housed in a temperature-controlled environment with a $12 \mathrm{~h}$ light/dark cycle at Department of Burns and Cutaneous Surgery, Xijing Hospital. All animal experiments were approved by and consistent with the principles of the Animal Research Ethics Board of Fourth Military Medical University, Xi'an, Shaanxi, China, as well as with local and national guidelines. Mice were transfected by injecting to caudal veins $60 \mu \mathrm{g} /$ mouse of plasmids pGMLV-ZsGreen1 or pGMLV-Klf4 in $60 \mu \mathrm{L}$ Micropolytransfector Tissue Reagent (Micropoly, \#MT215). Mice were challenged five days thereafter by peritoneal injection of $15 \mathrm{mg} / \mathrm{kg}$ lipopolysaccharide (Sigma, \#L2880), and cytokine levels in peritoneal sera or tissue were quantified $6 \mathrm{~h}$ after injection. Survival rate was monitored every $12 \mathrm{~h}$ for 2 days.

\section{Cells}

Mouse RAW264.7 macrophages were obtained from American Type Culture Collection (\#TIB-71), and cultured in RPMI 1640 (Corning Cellgro; \#10-040-CVR) containing $10 \%$ fetal bovine serum (Corning Cellgro; \#35-076-CV). Bone marrow-derived macrophages were isolated from male C57BL/C6 mice 6-8 weeks old, by culturing bone marrow for five days in RPMI 1640 emented with $10 \%$ fetal bovine serum, $20 \mathrm{ng} / \mathrm{mL}$ macrophage colony-stimulating factor (PeproTech; \#315-02), and $100 \mathrm{U} / \mathrm{mL}$ penicillin and streptomycin (Hyclone; \#SV30010).

\section{Plasmids and siRNAs}

pGMLV-ZsGreen1, pGMLV-Klf4, pGMR-TK, and pGL3-MCPIP1-Luc were purchased from Hegu Science $\&$ Technologies. Mouse MCPIP1 siRNAs were purchased from Biomics Biotechnologies, and used at $20 \mu \mathrm{M}$ by dilution in DEPC-treated water.

\section{Antibodies}

Antibodies to MCPIP1 (1:1, 000, \#25009-1-AP), Klf4 (1:500, \#11880-1-AP), H2AFY2 (1:1, 000, \#17030-1-AP), and $\alpha$-tubulin (1:1, 000,\#11224-1-AP) were purchased from Proteintech, along with mouse anti-rabbit IgG light chain (1:5, 000, \#SA00001-7). Anti-IKK $\gamma$ (phospho ser85) (1:1, 000, \#GTX32283) was purchased from GeneTex, while antibodies to IKK $\alpha / \beta$ (phospho Ser176/180) (1:1, 000, \#2697S), TAK1 (1:1, 000, \#5206), and TAK1 (phospho Thr184/187) (1:1, 000, \#4511) were procured from Cell Signaling Technology. Antibodies to K63-linked ubiquitin (1:1, 000, \#ab179434), TRAF6 (1:1, 000, \#ab94720), NF-кB

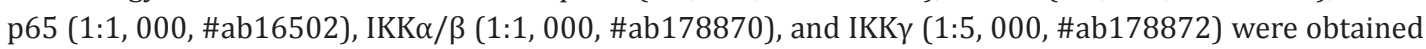
from Abcam. Rabbit polyclonal antibodies to GAPDH (1:1, 000, \#R1208-3), and goat anti-rabbit IgG (1:5, 000, \#EK020) were obtained from Zhuangzhi Biotech.

\section{Transient transfection}

RAW264.7 macrophages grown to 60-80 \% confluence were transfected for $24 \mathrm{~h}$ or $48 \mathrm{~h}$ with pGMLVZsGreen1, pGMLV-KIf4, pGMR-TK, or pGL3-MCPIP1-Luc in Opti-MEM (Thermo Gibco; \#31985062) and Lipofectamine 3000 Reagent (Thermo Invitrogen; \#L3000008), following the manufacturer's instructions. To silence MCPIP1, RAW264.7 macrophages grown to 60-80 \% confluence were transfected with siRNAs or scrambled siRNAs at a 1:1 v:v mixture with Micropoly-transfector cell reagent (Micropoly; \#MT110), following the manufacturer's instructions. After $48 \mathrm{~h}$, cells were stimulated with $1 \mu \mathrm{g} / \mathrm{mL}$ lipopolysaccharide, and analyzed for mRNA or protein levels of relevant molecules.

\section{Dual luciferase reporter assay}

RAW264.7 cells at 60-80 \% confluence were seeded in a 24-well plate, and co-transfected with pGL3MCPIP1-Luc, pGMLV-ZsGreen1 (NC) or pGMLV-Klf4 (Klf4), and pGMR-TK at a ratio of 10:9:1. After $24 \mathrm{~h}$, cells were washed with DPBS (Corning Cellgro; \#21-031-CVR) and lysed in passive lysis buffer. Luciferase activity was measured in the lysate by the Dual Luciferase Reporter Assay System (Promega; \#E1910) according to the manufacturer's instructions, and normalized to Renilla luciferase. Data were collected in triplicate from at least two experiments. 
Li et al.: KIf4 Alleviates Inflammation via Modulation of MCPIP1

\section{RNA isolation and quantitative real-time PCR}

Total RNA was extracted from cells or tissues using TRIzol Reagent (Thermo Invitrogen; \#15596-026) in accordance with the manufacturer's protocol. RNA extracts with OD260/280 ratio $2.0(0.5 \mathrm{mg})$ were reverse-transcribed by PrimeScript RT Master Mix (TaKaRa, \#RR036A) at $37^{\circ} \mathrm{C}$ for 15 minutes and at 85 ${ }^{\circ} \mathrm{C}$ for 5 seconds. Targets were then amplified in triplicate by quantitative RT-PCR using SYBR Green PCR Master Mix (TaKaRa; \#RR820A) on a Bio-Rad iQ5 Multicolor Real-Time PCR Detection System (Bio-Rad), normalized to GAPDH, and quantified by the comparative cycle threshold method $\left(2^{\triangle \Delta C T}\right)$. Primer sequences are listed in Table 1.

\section{Protein isolation and immunoblotting}

Cells were washed with ice-cold DPBS, lysed on ice for 30 minutes with RIPA buffer (HEART Biological Technology; \#WB009), and centrifuged at 12, 000 rpm for 10 minutes. Total protein was measured by BCA Protein Assay Kit (Thermo Scientific; \#23225), mixed with 5× SDS-PAGE loading buffer (HEART Biological Technology; \#R0993), and heated at $100{ }^{\circ} \mathrm{C}$ for 10 minutes. Samples ( $\left.40 \mu \mathrm{g}\right)$ were resolved by SDS-PAGE (HEART Biological Technology; \#WB005), and immunoblotted with corresponding antibodies.

\section{Extraction of nuclear and cytoplasmic proteins}

Cells were lysed using Nuclear and Cytoplasmic Protein Extraction Kit (Yeasen; \#20126ES50) in accordance with the manufacturer's protocol, and fractions were immunoblotted with appropriate antibodies.

\section{Immunoprecipitation}

Cells were lysed on ice for 30 minutes in Immunoprecipitation RIPA buffer (HEART Biological Technology; \#WB065) emented with protease inhibitor, centrifuged at 12, $000 \mathrm{rpm}$ for 10 minutes, and immunoprecipitated with Protein A/G PLUS-Agarose Immunoprecipitation Reagent (Santa Cruz; \#SC-2003) according to the manufacturer's instructions. Briefly, lysates were precleared by incubating on ice for 30 minutes with $1 \mu \mathrm{g}$ control IgG and $20 \mu \mathrm{L}$ Protein A/G PLUS-Agarose. Beads were pelleted by centrifugation at 2, $500 \mathrm{rpm}$ for 5 minutes at $4{ }^{\circ} \mathrm{C}$. The resulting supernatant (approximately 100-500 $\mu \mathrm{g}$ total protein) was incubated with $1 \mu \mathrm{g}$ primary antibody for $1 \mathrm{~h}$, and then with $20 \mu \mathrm{L}$ Protein A/G PLUS-Agarose overnight at 4 ${ }^{\circ} \mathrm{C}$. Immunoprecipitates were collected by centrifugation at $2,500 \mathrm{rpm}$ for 5 minutes at $4{ }^{\circ} \mathrm{C}$, washed 4 times with Immunoprecipitation RIPA buffer, resuspended in $40 \mu \mathrm{L} 1 \times$ loading buffer, and boiled for 10 minutes. Samples $(20 \mu \mathrm{L})$ were then resolved by SDS-PAGE and immunoblotted with appropriate antibodies.

Enzyme-linked immunosorbent assay (ELISA)

Mice were anesthetized by intraperitoneal injection of $1 \%$ pentobarbital sodium $(5 \mathrm{~mL} / \mathrm{kg})$. Blood was collected from the eyes, and centrifuged at 3, $000 \mathrm{rpm}$ for $10 \mathrm{~min}$. TNF- $\alpha$ (\#EMC102a.48) and IL-1 $\beta$ (\#EMC001b.48) in sera were quantified in triplicate by mouse ELISA kits according to the manufacturer's instructions (Neobioscience).

\section{Statistical analysis}

Data were analyzed in GraphPad Prism version 4.0 (GraphPad Software, La Jolla, CA, USA). Each experiment was repeated at least three times, and data are reported as mean \pm standard error of the

Table 1. Names and sequences of primers used for quantitative real-time-polymerase chain reaction

\begin{tabular}{llcc}
\hline Name & Species & Sense & Antisense \\
\hline TNF- $\alpha$ & Mouse & TATGGCCCAGACCCTCACA & GGAGTAGACAAGGTACAACCCATC \\
IL-1 $\beta$ & Mouse & CAACCAACAAGTGATATTCTCCATG & GATCCACACTCTCCAGCTGCA \\
IL-6 & Mouse & CAACGATGATGCACTTGCAGA & CTCCAGGTAGCTATGGTACTCCAGA \\
MCP-1 & Mouse & AGCAGCAGGTGTCCCAAAGA & GTGCTGAAGACCTTAGGGCAGA \\
Klf 4 & Mouse & GCATGTGCCCCAAGATTAAG & GTGACAGTCCCTGCTGTTCA \\
MCPIP1 & Mouse & CCACCTACCCATCCAGAGAG & GGGAAGACACCACACAGCTT \\
GAPDH & Mouse & TGTGTCCGTCGTGGATCTGA & TTGCTGTTGAAGTCGCAGGAG \\
\hline
\end{tabular}


mean. Statistical significance was determined by Student's $t$ test or one-way analysis of variance. Kaplan-Meier survival was assessed by log-rank test. A $P$ value less than 0.05 was considered statistically significant.

\section{Results}

Klf4 is an essential inhibitor of inflammation

As innate immune cells, macrophages represent the first line of defense. These cells are highly plastic, and acquire various phenotypes in response to environmental cues [5]. In mouse RAW264.7 and bone marrow-derived macrophages exposed to $1 \mu \mathrm{g} /$ $\mathrm{mL}$ lipopolysaccharide and characterized at $0 \mathrm{~h}, 0.5 \mathrm{~h}, 1 \mathrm{~h}$, 2 h, 6 h, 12 h, and 24 h (Fig. 1 A and $\mathrm{B})$, the pro-inflammatory cytokines $T N F-\alpha, I L-1 \beta, I L-6$, and $M C P-1$ were upregulated to various extents, peaking at about $2 \mathrm{~h}$ after stimulation. These data confirm that macrophages are activated and acquire a pro-inflammatory phenotype in response to lipopolysaccharides.

On the other hand, Klf4 plays importment roles in macrophage polarization and innate immunology regulation. However, the mechanisms by which Klf4 regulates inflammation are not fully understood. Strikingly, Klf4 mRNA and protein levels were significantly attenuated in RAW264.7 and bone marrow-derived macrophages exposed to lipopolysaccharides. Indeed, Klf4 expression reached the lowest levels about 2 to 6 hours after stimulation, and coincided with upregulation of pro-inflammatory genes (Fig. 1 A-D).

We then constructed a plasmid (pGMLV-Klf4) to overexpress Klf4 in RAW264.7 macrophages, with pGMLV-ZsGreen 1 as negative control (Fig. 1 E-G). As shown in Fig. 1 $\mathrm{H}$, activation of pro-inflammatory marker genes following exposure to lipopolysaccharide was significantly ameliorated in macrophages overexpressing Klf4, indicating strong antiinflammatory activity. These results collectively show that Klf4 expression diminishes in parallel to lipopolysaccharide-mediated activation of pro-inflammatory genes, while Klf4 overexpression blocks the effects of lipopolysaccharide.

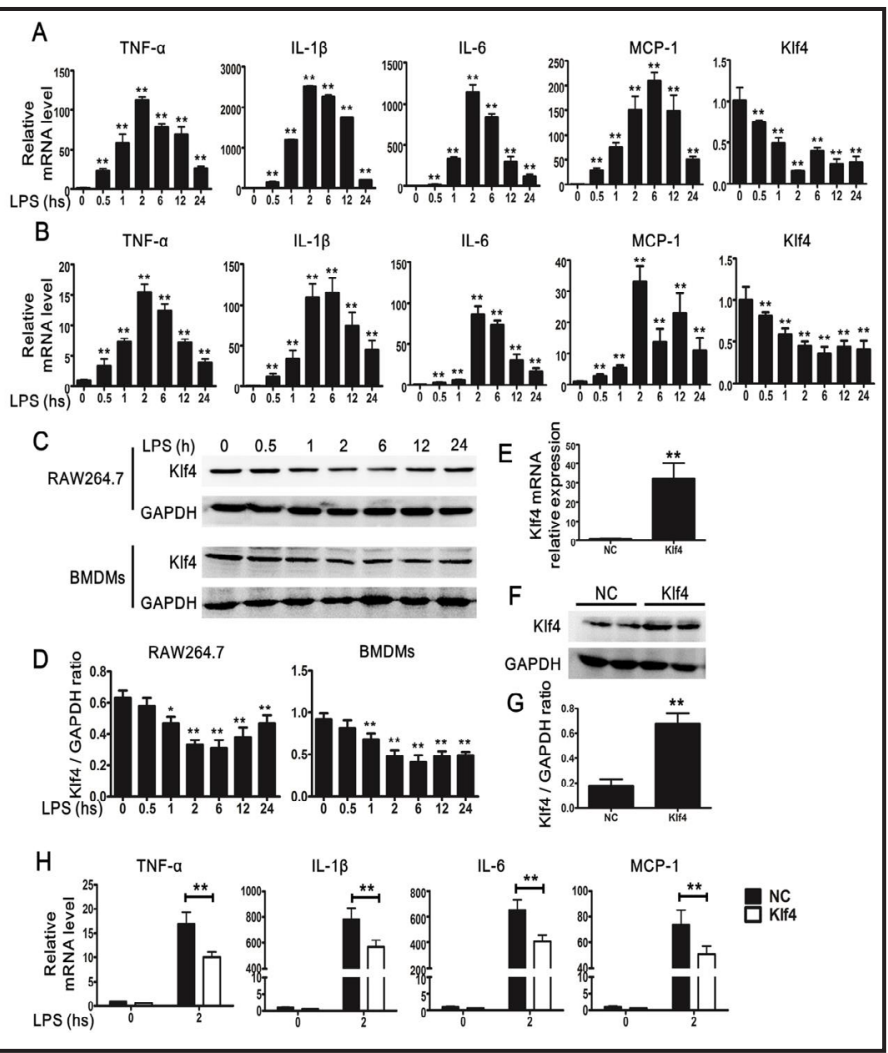

Fig. 1. Klf4 negatively regulated pro-inflammatory response in BMDMs and RAW264.7 macrophages upon stimulation with LPS. A and B. Inflammatory cytokines and Klf4 mRNA levels in (A) RAW264.7 macrophages and (B) BMDMs after different time points $(0 \mathrm{~h}, 0.5 \mathrm{~h}, 1 \mathrm{~h}, 2 \mathrm{~h}, 6 \mathrm{~h}, 12 \mathrm{~h}$ and $24 \mathrm{~h})$ of stimulation with LPS (1 $1 \mu \mathrm{g} /$ $\mathrm{ml}$ ). Gene expression levels were analyzed by quantitative real time PCR (qRT-PCR) and normalized to those in untreated cells. C and D. Klf4 protein levels after LPS treatment both in RAW264.7 macrophages and BMDMs, and normalized against GAPDH. E-G. Klf4 (E) mRNA levels and (F and G) protein levels in RAW264.7 macrophages after transient transfection of plasmid pGMLVGreen1 as negative control (NC) or pGMLV-Klf4 (Klf4) 48 h after transfection. H. Analysis of inflammatory cytokines transcripts by qRT-PCR following $2 \mathrm{~h}$ LPS stimulation in RAW264.7 macrophages transfected with NC or Klf4, and normalized against GAPDH. Data are shown as mean \pm SEM. ${ }^{*} \mathrm{p}<0.05,{ }^{* *} \mathrm{p}<0.01$ vs. negative control. Data are representative of at least three independent experiments. 
Nuclear Klf4 suppresses cytoplasmic NF- $\kappa B$ signaling

Furthermore, we found that phosphorylation of molecules in the NF- $\kappa \mathrm{B}$ pathway, including TAK1, IKK- $\alpha / \beta$, and IKK- $\gamma$, was significantly enhanced in macrophages treated with $1 \mu \mathrm{g} / \mathrm{mL}$ lipopolysaccharide for various durations. In line with this result, expression of K63linked polyubiquitins was also enhanced (Fig. 2 A and B). Strikingly, activation of these kinases in response to lipopolysaccharide was less pronounced in RAW264.7 macrophages transiently transfected for $48 \mathrm{~h}$ with pGMLV-Klf4 to overexpress Klf4 (Fig. 2 B). We note that bone marrow-derived macrophages were inefficiently transfected, and were thus unsuitable for similar analysis.

Klf4 accumulated exclusively in the nucleus under basal conditions or under lipopolysaccharide stimulation, while IKK complexes and TAK1 were cytoplasmic. Further, Klf4 expression reduced the translocation of $\mathrm{p} 65$ to the nucleus following exposure to lipopolysaccharide (Fig. 2 C). Collectively, these results indicate that Klf4 ameliorates NF- $\mathrm{KB}$ signaling only indirectly.

\section{Klf4 mediates MCPIP1}

transcription to regulate $N F-\kappa B$

The deubiquitinase MCPIP1 inhibits NF- $\kappa B$ activation, and is driven by a promoter that contains multiple Klf4 sites [32]. As shown in Fig. $3 \mathrm{~A}$ to $\mathrm{C}$, transcript and protein levels of MCPIP1 were both elevated in RAW264.7 macrophages transiently overexpressing Klf4, implying that Klf4 boosts MCPIP1 expression. Similarly, RAW264.7 macrophages transfected with a dual luciferase reporter vector under the control of the MCPIP1 promoter, expressed luciferase only upon Klf4 overexpression (Fig. 3 D). Moreover, immunoblotting of subcellular fractions revealed that MCPIP1 accumulates both in the nucleus and cytoplasm, and is upregulated upon Klf4 overexpression under both basal conditions and under lipopolysaccharide stimulation. Klf4 overexpression also reduced p65 translocation to the nucleus following exposure to lipopolysaccharide (Fig. $3 \mathrm{E}$ and F). Together, these results indicate that Klf4 regulates the expression of MCPIP1, which then translocates to the cytoplasm to regulate NF- $\kappa B$.

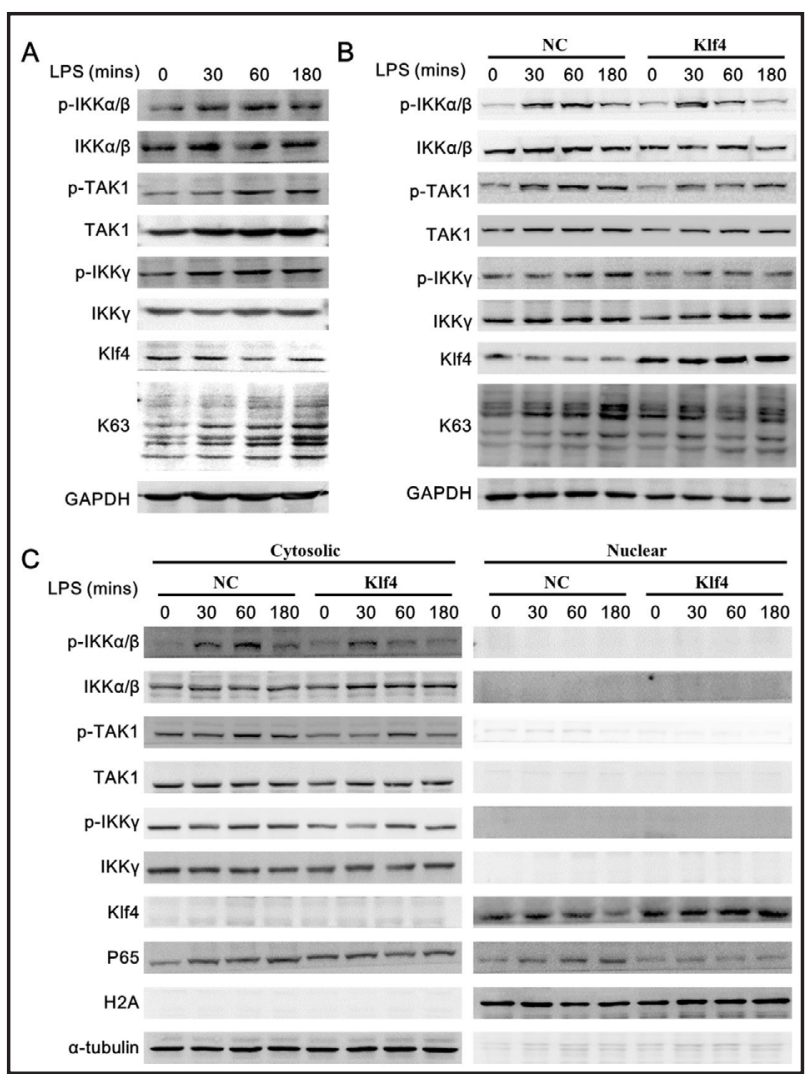

Fig. 2. Klf4 accumulates in nucleus and negatively regulates NF- $\kappa$ B pathway upon LPS stimuli. A. Immunoblotting analysis of p-TAK1, TAK1, p-IKK $\alpha / \beta$, IKK $\alpha / \beta, \mathrm{p}-\mathrm{IKK} \gamma, \mathrm{IKK} \gamma, \mathrm{Klf} 4$ and K63 polyubiquitin chains expression in BMDMs either uninfected or infected with LPS for indicated time points, and GAPDH as loading control. B. NC and Klf4 overexpressed Raw264.7 macrophages either unstimulated or stimulated with LPS for indicated time points were analyzed by immunoblotting for $\mathrm{p}$-TAK1, TAK1, p-IKK $\alpha / \beta$, IKK $\alpha / \beta$, $\mathrm{p}-\mathrm{IKK} \gamma, \mathrm{IKK} \gamma, \mathrm{Klf4}, \mathrm{K} 63$ polyubiquitin chains, and GAPDH as loading control. C. Cytoplasmic and nuclear extracts of NC and Klf4 overexpressed Raw264.7 macrophages that either unstimulated or stimulated with LPS for indicated time points were analyzed by immunoblotting for $\mathrm{p}$-TAK1, TAK1, p-IKK $\alpha / \beta, I K K \alpha / \beta, \mathrm{p}-\mathrm{IKK} \gamma, \mathrm{IKK} \gamma, \mathrm{Klf} 4, \mathrm{P} 65, \mathrm{H} 2 \mathrm{~A}$ and $\alpha$-tubulin. Purity of cytoplasmic and nuclear fraction is depicted by $\alpha$-tubulin and H2A as loading control. Data are representative of at least three independent experiments. 
MCPIP1 acts as a negative regulator in the inflammatory response via modulation of $N F-\kappa B$ signaling

MCPIP1 is widely reported to suppress the inflammatory response by deubiquitinating inflammatory proteins [12, 24]. In line with this model, we found that exposure to lipopolysaccharide dramatically enhanced MCPIP1 expression in macrophages, as shown in Figure $4 \mathrm{~A}$ to $\mathrm{C}$, especially at 2 to 6 hours after exposure. We then synthesized three small interfering RNAs against MCPIP1 (siMCPIP1), of which siMCPIP1-2 and siMCPIP1-3 markedly reduced MCPIP1 expression after transient transfection for 48 $\mathrm{h}$, in comparison to scrambled siRNA (siNC). In cells transfected with siMCPIP1-3 for $48 \mathrm{~h}$ (Fig. $4 \mathrm{D}$ to $\mathrm{F}$ ), the pro-inflammatory cytokines TNF- $\alpha$, IL-1 $\beta$, IL- 6 , and MCP-1 were notably upregulated at mRNA levels both in normal conditions and in response to lipopolysaccharide (Fig. 4 G), suggesting that MCPIP1 knockdown elicits hyperinflammation. In addition, IKK complexes, TAK1, and K63linked polyubiquitins were elevated to various extents in macrophages transfected with siMCPIP1-3 for $48 \mathrm{~h}$ and then challenged with $1 \mu \mathrm{g} / \mathrm{mL}$ lipopolysaccharide for $0,30,60$, and $180 \mathrm{~min}$ (Fig. $4 \mathrm{H}$ ). Taken together, these results indicate

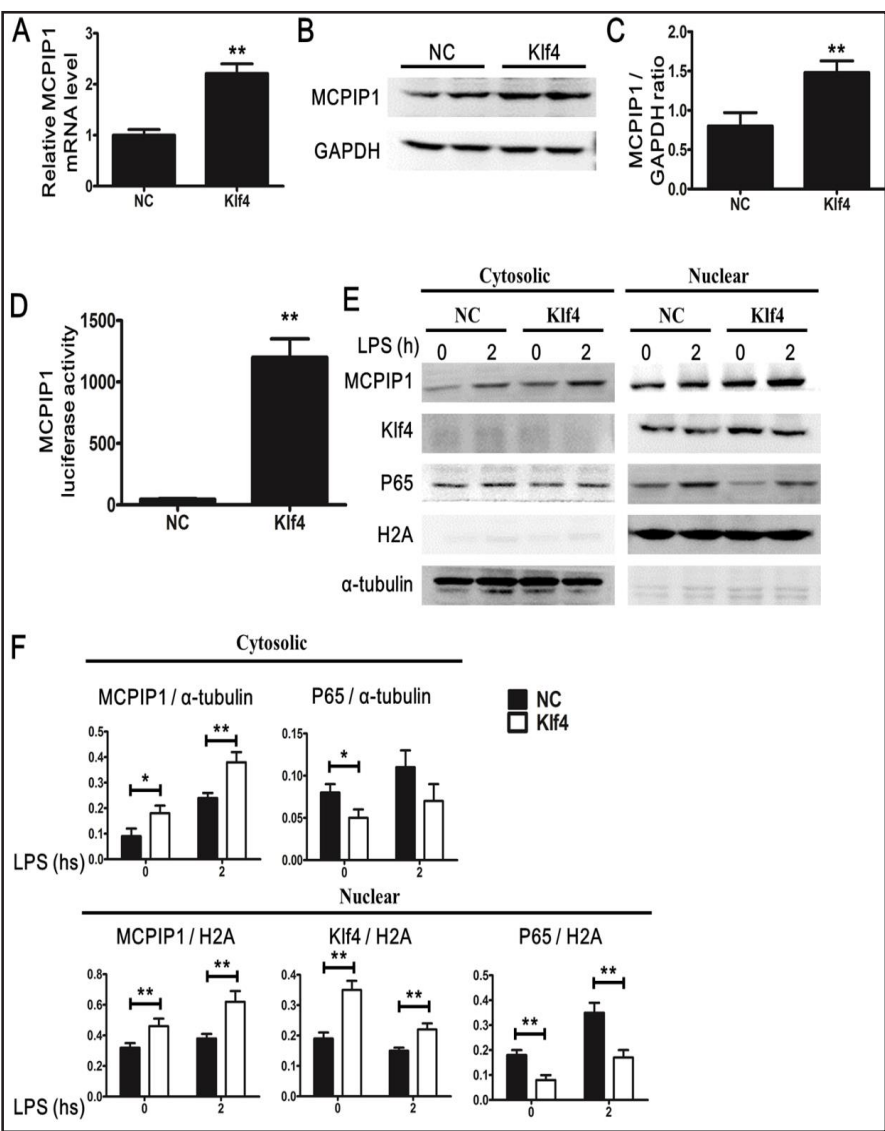

Fig. 3. Klf4 induced MCPIP1 transcription to regulate NF- $\mathrm{kB}$ activation. A-C. MCPIP1 (A) mRNA levels and (B and C) protein levels in RAW264.7 macrophages after transient transfection of plasmid pGMLV-Green1 as negative control (NC) or pGMLV-Klf4 (Klf4) for $48 \mathrm{~h}$, and normalized against GAPDH. D. RAW264.7 macrophages were cotransfected with MCPIP1 promoter luciferase reporter vector pGL3-MCPIP1-Luc, pGMLV-Green1 (NC)/ pGMLV-Klf4 (Klf4) and pGMR-TK at ratio 10:9:1 for $24 \mathrm{~h}$, and luciferase activity was measured in the lysate by the Dual-luciferase reporter Assay System and normalized to Renilla luciferase assay. Overxpression of Klf4 enhanced MCPIP1 luciferase reporter activity. E and F. Cytoplasmic and nuclear extracts of NC and Klf4 overexpressed Raw264.7 macrophages that either uninfected or infected with LPS for $2 \mathrm{~h}$ were analyzed by immunoblotting for MCPIP1, Klf4, P65, $\mathrm{H} 2 \mathrm{~A}$ and $\alpha$-tubulin. Purity of cytoplasmic and nuclear fraction is depicted by $\alpha$-tubulin and H2A as loading control. Data are shown as mean \pm SEM. ${ }^{*} \mathrm{p}<0.05,{ }^{* *} \mathrm{p}<0.01$ vs. negative control. Data are representative of at least three independent experiments. that MCPIP1 suppresses the inflammatory response to lipopolysaccharide by modulating NF- $\kappa$ B signaling.

\section{Klf4 indirectly inactivates TRAF6 via MCPIP1}

Recent studies identified TRAF proteins as essential in the response to lipopolysaccharide. For example, binding of lipopolysaccharide to TLR4 triggers recruitment of MyD88 and IRAK1/4, which subsequently recruit TRAF6 to initiate downstream signaling [6]. In RAW264.7 macrophages transfected with negative control or Klf4 plasmid for $48 \mathrm{~h}$ and then 
stimulated with lipopolysaccharide, we found by immunoprecipitation that Klf4 coprecipitates with MCPIP1, but not with TRAF6 or K63linked polyubiquitins (Fig. 5 A). Moreover, we found that TRAF6 coprecipitates with K63 polyubiquitins and MCPIP1, but not with Klf4, and that accumulation of K63 polyubiquitins was lower in cells overexpressing Klf4 (Fig. 5 B). Moreover, pulldown experiments showed that cytoplasmic MCPIP1 was in complex with TRAF6 and K63 polyubiquitins, but not with Klf4. On the other hand, nuclear MCPIP1 was associated with Klf4, but not TRAF6 and K63 polyubiquitins (Fig. $5 \mathrm{C}$ ). Together, these data imply that increased Klf4 expression elevates MCPIP1 activity in the nucleus to attenuate TRAF 6 deubiquitination in the cytoplasm, and thereby suppress NF- $\kappa \mathrm{B}$ signaling in response to lipopolysaccharide (Fig. 7).

Klf4 overexpression in vivo alleviates inflammation in septic mice

The role of Klf4 in inflammation was investigated in vivo using mice transfected for five days with pGMLVZsGreen1 or pGMLV-KIf4 by injection through the caudal vein. These mice were then treated with $15 \mathrm{mg} / \mathrm{kg}$ lipopolysaccharide by intraperitoneal injection. We found that survival $48 \mathrm{~h}$ after exposure was higher in mice overexpressing Klf4 (approximately $70 \%$ ) than in mice transfected with pGMLV-Green1 (approximately $40 \%$, Fig. 6 A). Moreover, secretion of inflammatory cytokines into sera, including TNF- $\alpha$ and IL-1 $\beta$, was wellcontrolled in mice overexpressing Klf4 6 h after exposure to lipopolysaccharide (Fig. 6 B). Analysis of the liver, lungs, and kidney, which are the organs most susceptible to septic injury, indicated that although Klf4 mRNA levels in the kidney were comparable among groups, levels in the liver and lungs were > 200- and 15-fold higher in mice overexpressing Klf4 than in control mice (Fig. 6C), regardless of exposure to lipopolysaccharide. However, lipopolysaccharide-induced expression of pro-inflammatory mRNAs in the liver, including TNF- $\alpha$, IL-1 $\beta$,

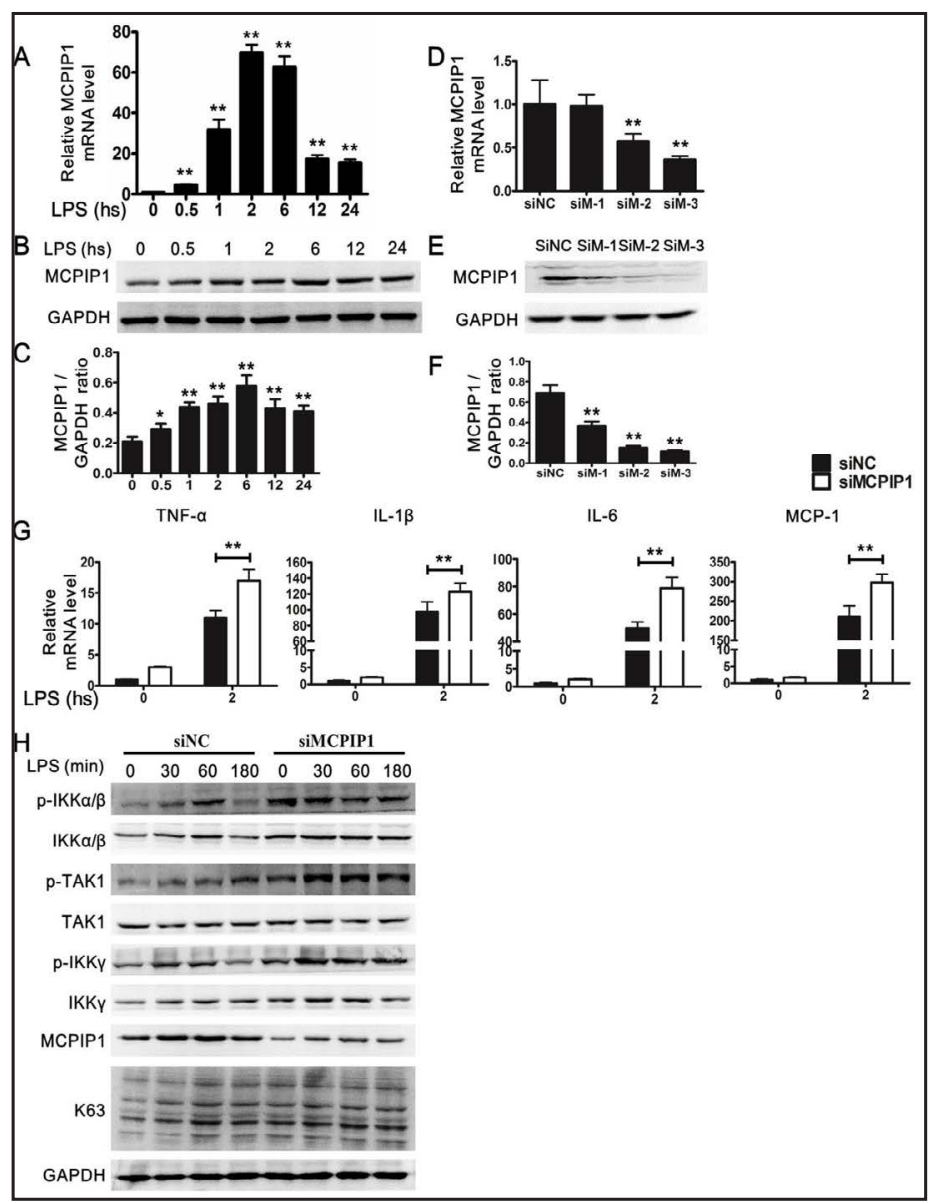

Fig. 4. A-C. MCPIP1 (A) mRNA levels and (B and C) protein levels in RAW264.7 macrophages after different points of stimulation with LPS $(1 \mu \mathrm{g} / \mathrm{ml})$, and normalized against GAPDH. D-F. Gene silencing effects of three different siRNA fragments targeting MCPIP1 (siMCPIP1) on RAW264.7 macrophages through qRT-PCR (D) and immunoblotting (E and F) analysis respectively. G. Analysis of inflammatory cytokines transcripts by qRT-PCR followed by $2 \mathrm{~h}$ LPS stimulation in RAW264.7 macrophages transfected with siNC or siMCPIP1. H. RAW264.7 macrophages silenced MCPIP1 either unstimulated or stimulated with LPS for indicated time points were analyzed by immunoblotting for $\mathrm{p}$-TAK1, TAK1, $\mathrm{p}-\mathrm{IKK} \alpha / \beta, \mathrm{IKK} \alpha / \beta, \mathrm{p}-\mathrm{IKK} \gamma, \mathrm{IKK} \gamma, \mathrm{MCPIP} 1, \mathrm{~K} 63$ polyubiquitin chains, and GAPDH as loading control. Data are shown as mean \pm SEM. ${ }^{*} \mathrm{p}<0.05,{ }^{* *} \mathrm{p}<0.01$ vs. negative control. Data are representative of at least three independent experiments. 
Fig. 5. Klf4 indirectly deubiquitinates K63 poly ubiquitins on TRAF6 via regulation of MCPIP1. A and B. RAW264.7 macrophages transfected NC or Klf4 either unstimulated or stimulated with LPS for $2 \mathrm{~h}$ were immunoprecipitated with anti-Klf4, anti-TRAF6 or IgG control. Pulldowns and corresponding wholecell lysates were then immunoblotted for MCPIP1, Klf4 TRAF6, K63 poly ubiquitins and GAPDH (loading control). C. Cytoplasmic and nuclear extracts of NC and Klf4 overexpressed RAW264.7 macrophages that either uninfected or infected with LPS for 2 $\mathrm{h}$ were immunoprecipitated with antiMCPIP1 or IgG control. Pulldowns and corresponding Cytoplasmic and nuclear lysates were then immunoblotted for MCPIP1, Klf4 TRAF6, K63 poly ubiquitins, $\mathrm{H} 2 \mathrm{~A}$ and $\alpha$-tubulin. Purity of cytoplasmic and nuclear fraction is depicted by $\alpha$-tubulin and H2A loading control. Data are representative of at least three independent experiments.

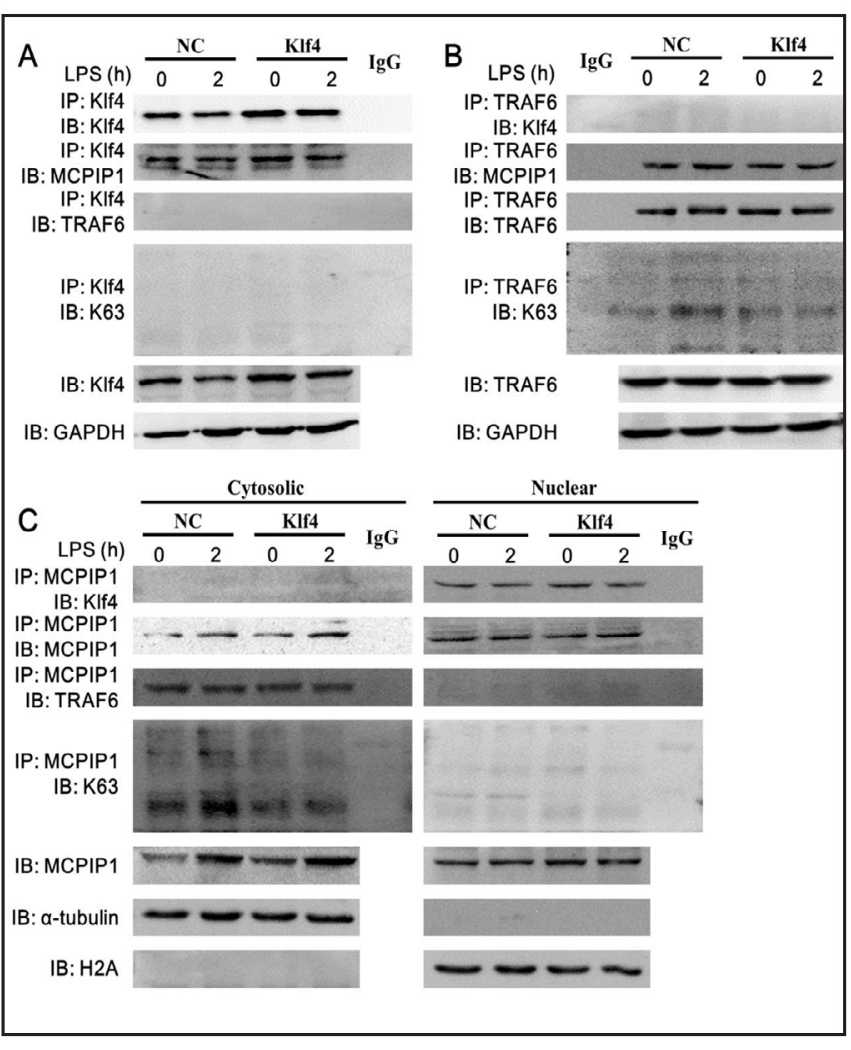

Fig. 6. Klf4 overexpression ameliorates inflammation in LPSinduced septic mice. A. C57BL/ C6 mice were transfected pGMLVGreen1 (NC, $\mathrm{n}=10$ ) or pGMLVKlf4 (Klf4, $n=10$ ) through injection of caudal veins for 5 days then inoculated via intraperitoneal route with saline or LPS (15 mg/ $\mathrm{kg}$ ) to observe their morbidity. The mice survival is represented in a Kaplan-Meier plot. Statistical analysis on survival curves were performed using log-rank test. B. mice transfected with NC $(n=5)$ or Klf4 ( $n=5)$ were inoculated via intraperitoneal route with saline or LPS (15 mg/kg). $6 \mathrm{~h}$ later, sera were analyzed by enzyme-linked immuno sorbent assay (ELISA) for TNF- $\alpha$ and IL-1 $\beta$ levels. C. qRTPCR analysis of transcript for Klf4

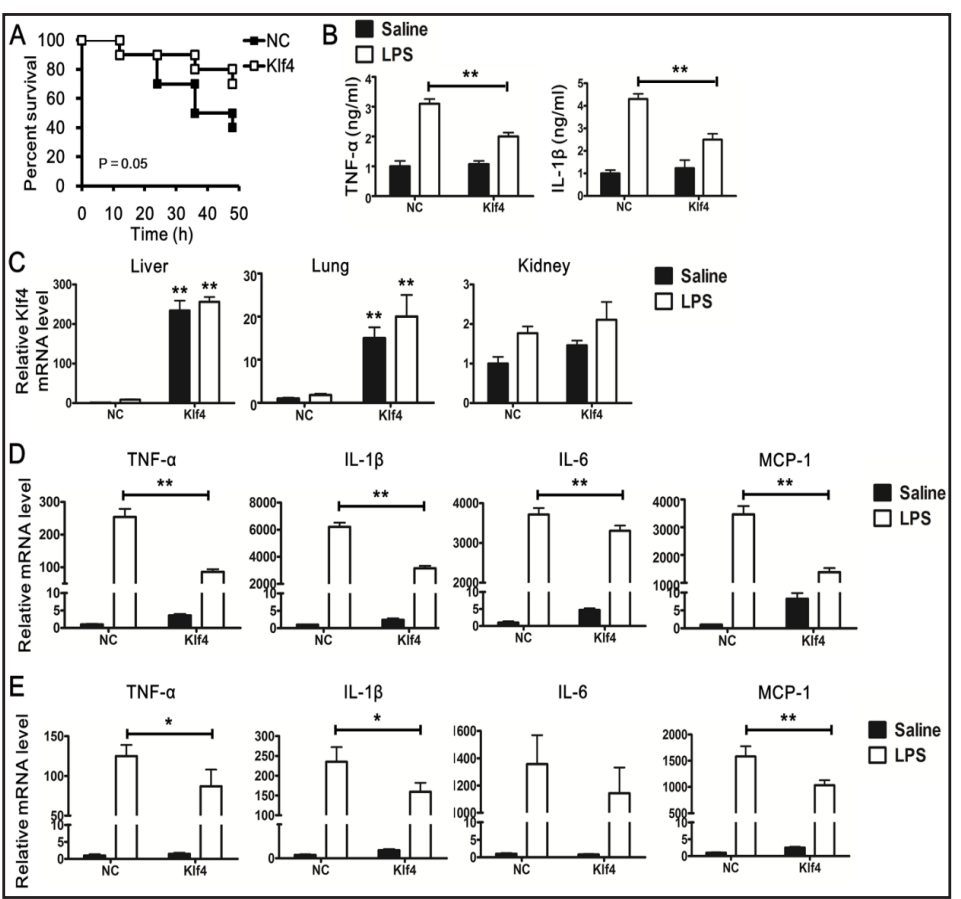
gene in livers, lungs and kidneys of mice transfected with NC $(n=6)$ or Klf4 $(n=6) 6 \mathrm{~h}$ after inoculation of LPS. D and E. qRT-PCR analysis of transcripts for inflammatory cytokines TNF- $\alpha$, IL-1 $\beta$, IL- 6 and MCP-1 genes in livers (D) and lungs (E) of mice transfected with NC $(n=6)$ and Klf4 $(n=6) 6 \mathrm{~h}$ after inoculation of LPS. Data are shown as mean \pm SEM. ${ }^{*} \mathrm{p}<0.05,{ }^{* *} \mathrm{p}<0.01$ vs. negative control. 
IL-6, and MCP-1, was remarkably lower in the former (Fig. 6D). Similar results, except IL-6, were observed in the lungs (Fig. $6 \mathrm{E}$ ), but not in the kidney (data not shown). Together, these data show that Klf4 is an important inhibitor of the inflammatory response, and partially protects mice from lipopolysaccharide-induce sepsis.

\section{Discussion}

Infection by bacteria, viruses, fungi, parasites, and other pathogens elicit local and systemic inflammatory responses [37, 38], of which sepsis is a leading cause of mortality in adults and children $[39,40]$. Since inflammation and innate immunity against bacteria are hallmarks of sepsis [41, 42], enhancing the latter while mitigating the former is a worthwhile therapeutic strategy [43]. One possibility is to exploit Klf4, a zinc-finger transcription factor that was shown to either promote $[44,45]$ or inhibit inflammatory signaling $[33,46]$. In light of these contradictory results, we now present strong evidence that Klf4 overexpression, at least under our experimental conditions, significantly suppresses the inflammatory response of macrophages to lipopolysaccharide, markedly decreasing the release of pro-inflammatory cytokines such as TNF- $\alpha$, IL-1 $\beta$, IL-6, and MCP- 1 . Nevertheless, further in vivo experiments in animals and even in humans are still needed to conclusively demonstrate that Klf4 is anti-inflammatory.

Importantly, Klf4 appears to inhibit lipopolysaccharide-induced inflammation via NF$\kappa B$, as evidenced by a dramatic decrease in phosphorylation of TAK1, IKK $\alpha / \beta$, and IKK $\gamma$, as well as in the expression of K63 polyubiquitin chains. However, we also found that while the affected proteins are cytoplasmic, Klf4 is nuclear. Immunoprecipitation also indicated that Klf4 did not bind any of the affected proteins, implying that Klf4 does not directly regulate NF- $\kappa$ B effector proteins. Subsequently, we found the Klf4 avidly binds MCPIP1, which, in turn interacts with a protein upstream to NF- $\mathrm{KB}$ such as TRAF6. Indeed, MCPIP1 was previously shown to inhibit lipopolysaccharide-induced JNK and NF- $\mathrm{KB}$ signaling by deubiquitinating TRAF proteins $[2,12,22]$.

Strikingly, MCPIP1 is itself induced during inflammation [32], and by inflammatory signals such as NF-KB, IL-1b, TNF-a, and IL-6 $[8,27]$, although it is not clear how MCPIP1 is regulated, or whether it provides negative feedback regulation. We believe that such a feedback mechanism is likely. Indeed, we observed that Klf4 overexpression also upregulates MCPIP1 both at the mRNA and protein level, and that Klf4 stimulates expression of a luciferase reporter gene controlled by the MCPIP1 promoter. In addition, our data also indicate that loss of MCPIP1 significantly enhances the release of the pro-inflammatory cytokines TNF- $\alpha$, IL-1 $\beta$, IL-6, and MCP-1, reinforcing that notion that MCPIP1 is anti-inflammatory. Therefore, Klf4 appears to modulate lipopolysaccharide-induced inflammation via MCPIP1.

Inline with invitro data, mice overexpressing Klf4 wereless sensitivetolipopolysaccharide, secreting lower levels of pro-inflammatory cytokines into sera, including TNF- $\alpha$ and IL-1 $\beta$, in response to exposure. Most importantly, Klf4 overexpression ameliorated the severity of 
clinical symptoms (data not shown) and reduced mortality. However, the protective effects of Klf4 were observed only in the lungs and liver, but not in the kidney, even though all three are severely damaged during sepsis. This result correlates with Klf4 levels, which were variable in these tissues perhaps as a result of variable dissemination of the plasmid. For example, we found that Klf4 overexpression was much stronger in the liver than in the lungs and kidney of the same mice, as was previously observed [47]. In addition, specific cellular conditions or greater reparative capacity in the liver may enhance the effects of Klf4. In any case, these observations suggest that the protective effects of Klf4 against inflammation may differ depending on tissue type.

Some major issues remain to be addressed. For example, why is Klf4 suppressed in response to lipopolysaccharide? One possibility is that miR-7 or other factors control Klf4 expression to modulate physiological process in macrophages, which are under study in our group. The mechanisms underlying MCPIP1 upregulation but Klf4 suppression response to lipopolysaccharide should also be clarified. On the other hand, MCPIP1 may also modify Klf4 ubiquitination, and thereby alter its regulatory effects on cellular processes such as cell growth, proliferation, and differentiation [48, 49]. Indeed, Klf4 sumoylation promotes IL4-induced macrophage M2 polarization [50]. Finally, the long-term effects of Klf4 against lipopolysaccharide-induced inflammation remain unknown.

Taken together, the data demonstrate that Klf4 modulates lipopolysaccharide-induced inflammation and tissue damage both in vivo and in vitro by suppressing the release of proinflammatory cytokines, inducing MCPIP1, and inhibiting NF- $\mathrm{KB}$ signaling. Our findings not only provide strong evidence that Klf4 is involved in innate immunity and sepsis, but also highlight new research opportunities into the role of Klf4 in various inflammatory processes and disorders, as well as into its potential as therapeutic target.

\section{Acknowledgements}

This work was funded by grants from the National Natural Science Foundation of China (No.81530064).

\section{Disclosure Statement}

The authors declare that the research was conducted in the absence of any commercial or financial relationships that could be construed as a potential Disclosure Statement.

\section{References}

1 Henneke P, Golenbock DT: Phagocytosis, innate immunity, and host-pathogen specificity. J Exp Med 2004;199:1-4.

-2 Liang J, Saad Y, Lei T, Wang J, Qi D, Yang Q Kolattukudy PE, Fu M: MCP-induced protein 1 deubiquitinates TRAF proteins and negatively regulates JNK and NF-kappaB signaling. J Exp Med 2010;207:2959-2973.

-3 Medzhitov R, Preston-Hurlburt P, Janeway CJ: A human homologue of the Drosophila Toll protein signals activation of adaptive immunity. Nature 1997;388:394-397.

-4 Rock KL, Latz E, Ontiveros F, Kono H: The sterile inflammatory response. Annu Rev Immunol 2010;28:321342.

5 Huang S, Miao R, Zhou Z, Wang T, Liu J, Liu G, Chen YE, Xin HB, Zhang J, Fu M: MCPIP1 negatively regulates toll-like receptor 4 signaling and protects mice from LPS-induced septic shock. Cell Signal 2013;25:12281234.

6 Baker RG, Hayden MS, Ghosh S: NF-kappaB, inflammation, and metabolic disease. Cell Metab 2011;13:1122. 


\section{Cellular Physiology Cell Physiol Biochem 2018;47:2278-2290

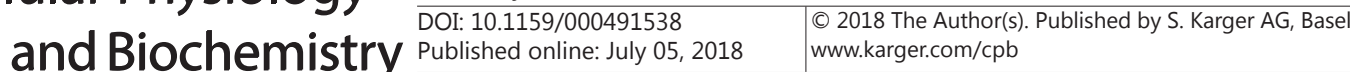

7 Karin M, Greten FR: NF-kappaB: Linking inflammation and immunity to cancer development and progression. Nat Rev Immunol 2005;5:749-759.

8 Liang J, Wang J, Azfer A, Song W, Tromp G, Kolattukudy PE, Fu M: A novel CCCH-zinc finger protein family regulates proinflammatory activation of macrophages. J Biol Chem 2008;283:6337-6346.

-9 Ma J, Chen T, Mandelin J, Ceponis A, Miller NE, Hukkanen M, Ma GF, Konttinen YT: Regulation of macrophage activation. Cell Mol Life Sci 2003;60:2334-2346.

10 Beutler B, Rietschel ET: Innate immune sensing and its roots: The story of endotoxin. Nat Rev Immunol 2003;3:169-176.

11 Dong W, Liu Y, Peng J, Chen L, Zou T, Xiao H, Liu Z, Li W, Bu Y, Qi Y: The IRAK-1-BCL10-MALT1-TRAF6-TAK1 cascade mediates signaling to NF-kappaB from Toll-like receptor 4. J Biol Chem 2006;281:26029-26040.

12 Jura J, Skalniak L, Koj A: Monocyte chemotactic protein-1-induced protein-1 (MCPIP1) is a novel multifunctional modulator of inflammatory reactions. Biochim Biophys Acta 2012;1823:1905-1913.

13 Sato S, Sanjo H, Takeda K, Ninomiya-Tsuji J, Yamamoto M, Kawai T, Matsumoto K, Takeuchi O, Akira S: Essential function for the kinase TAK1 in innate and adaptive immune responses. Nat Immunol 2005;6:1087-1095.

14 Pickart CM, Fushman D: Polyubiquitin chains: Polymeric protein signals. Curr Opin Chem Biol 2004;8:610616.

15 Panda S, Nilsson JA, Gekara NO: Deubiquitinase MYSM1 regulates innate immunity through inactivation of TRAF3 and TRAF6 complexes. Immunity 2015;43:647-659.

-16 Komander D, Rape M: The ubiquitin code. Annu Rev Biochem 2012;81:203-229.

17 Ikeda F, Crosetto N, Dikic I: What determines the specificity and outcomes of ubiquitin signaling? Cell 2010;143:677-681.

18 Weissman AM, Shabek N, Ciechanover A: The predator becomes the prey: Regulating the ubiquitin system by ubiquitylation and degradation. Nat Rev Mol Cell Biol 2011;12:605-620.

19 Dikic I, Wakatsuki S, Walters KJ: Ubiquitin-binding domains - from structures to functions. Nat Rev Mol Cell Biol 2009;10:659-671.

20 Lopez-Castejon G, Edelmann MJ: Deubiquitinases: Novel therapeutic targets in immune surveillance? Mediators Inflamm 2016;2016:3481371.

21 Aksentijevich I, Zhou Q: NF-kappaB pathway in autoinflammatory diseases: Dysregulation of protein modifications by ubiquitin defines a new category of autoinflammatory diseases. Front Immunol 2017;8:399.

22 Sun SC: Deubiquitylation and regulation of the immune response. Nat Rev Immunol 2008;8:501-511.

23 Cifuentes RA, Cruz-Tapias P, Rojas-Villarraga A, Anaya JM: ZC3H12A (MCPIP1): Molecular characteristics and clinical implications. Clin Chim Acta 2010;411:1862-1868.

24 Fu M, Blackshear PJ: RNA-binding proteins in immune regulation: A focus on CCCH zinc finger proteins. Nat Rev Immunol 2017;17:130-143.

25 Zhou L, Azfer A, Niu J, Graham S, Choudhury M, Adamski FM, Younce C, Binkley PF, Kolattukudy PE: Monocyte chemoattractant protein-1 induces a novel transcription factor that causes cardiac myocyte apoptosis and ventricular dysfunction. Circ Res 2006;98:1177-1185.

-26 Liang J, Song W, Tromp G, Kolattukudy PE, Fu M: Genome-wide survey and expression profiling of CCCHzinc finger family reveals a functional module in macrophage activation. Plos One 2008;3:e2880.

-27 Skalniak L, Mizgalska D, Zarebski A, Wyrzykowska P, Koj A, Jura J: Regulatory feedback loop between NFkappaB and MCP-1-induced protein 1 RNase. Febs J 2009;276:5892-5905.

-28 Matsushita K, Takeuchi O, Standley DM, Kumagai Y, Kawagoe T, Miyake T, Satoh T, Kato H, Tsujimura T, Nakamura H, Akira S: Zc3h12a is an RNase essential for controlling immune responses by regulating mRNA decay. Nature 2009;458:1185-1190.

29 Mizgalska D, Wegrzyn P, Murzyn K, Kasza A, Koj A, Jura J, Jarzab B, Jura J: Interleukin-1-inducible MCPIP protein has structural and functional properties of RNase and participates in degradation of IL-1beta mRNA. Febs J 2009;276:7386-7399.

-30 Li M, Cao W, Liu H, Zhang W, Liu X, Cai Z, Guo J, Wang X, Hui Z, Zhang H, Wang J, Wang L: MCPIP1 downregulates IL-2 expression through an ARE-independent pathway. Plos One 2012;7:e49841.

-31 Xu J, Fu S, Peng W, Rao Z: MCP-1-induced protein-1, an immune regulator. Protein Cell 2012;3:903-910. 


\section{Cellular Physiology Cell Physiol Biochem 2018;47:2278-2290 \begin{tabular}{l|l} 
DOI: 10.1159/000491538 & $\begin{array}{l}\text { O 2018 The Author(s). Published by S. Karger AG, Basel } \\
\text { www.karger.com/cpb }\end{array}$
\end{tabular}}

32 Kapoor N, Niu J, Saad Y, Kumar S, Sirakova T, Becerra E, Li X, Kolattukudy PE: Transcription factors STAT6 and KLF4 implement macrophage polarization via the dual catalytic powers of MCPIP. J Immunol 2015;194:6011-6023.

33 Liao X, Sharma N, Kapadia F, Zhou G, Lu Y, Hong H, Paruchuri K, Mahabeleshwar GH, Dalmas E, Venteclef N, Flask CA, Kim J, Doreian BW, Lu KQ, Kaestner KH, Hamik A, Clement K, Jain MK: Kruppel-like factor 4 regulates macrophage polarization. J Clin Invest 2011;121:2736-2749.

-34 Zhu LF, Chen QR, Chen SZ, Wang LY, Luo XF, Ren JH, Yuan XH, Wu XQ, Zeng YL, Xiao M, Chen YQ Chen YY, Lin $\mathrm{MH}, \mathrm{Wu} \mathrm{ZJ}$, Chen ZZ, Hu JD, Yang T: The construction and identification of induced pluripotent stem cells derived from acute myelogenous leukemia cells. Cell Physiol Biochem 2017;41:1661-1674. Ghaleb AM, Yang VW: Kruppel-like factor 4 (KLF4): What we currently know. Gene 2017;611:27-37. Liang S, Yu H, Chen X, Shen T, Cui Z, Si G, Zhang J, Cheng Y, Jia S, Song S, Zhang X, Yu X: PDGF-BB/KLF4/VEGF signaling axis in pulmonary artery endothelial cell angiogenesis. Cell Physiol Biochem 2017;41:2333-2349.

37 Nunez LO, Cambiaso-Daniel J, Branski LK, Norbury WB, Herndon DN: Predicting and managing sepsis in burn patients: Current perspectives. Ther Clin Risk Manag 2017;13:1107-1117.

-38 Kopitar-Jerala N: The role of interferons in inflammation and inflammasome activation. Front Immunol 2017;8:873.

39 Williams FN, Herndon DN, Hawkins HK, Lee JO, Cox RA, Kulp GA, Finnerty CC, Chinkes DL, Jeschke MG: The leading causes of death after burn injury in a single pediatric burn center. Crit Care 2009;13:R183.

-40 D’Avignon LC, Hogan BK, Murray CK, Loo FL, Hospenthal DR, Cancio LC, Kim SH, Renz EM, Barillo D, Holcomb JB, Wade CE, Wolf SE: Contribution of bacterial and viral infections to attributable mortality in patients with severe burns: An autopsy series. Burns 2010;36:773-779.

41 Cohen J: The immunopathogenesis of sepsis. Nature 2002;420:885-891.

42 Savva A, Roger T: Targeting toll-like receptors: Promising therapeutic strategies for the management of sepsis-associated pathology and infectious diseases. Front Immunol 2013;4:387.

43 Fang H, Jiang W, Cheng J, Lu Y, Liu A, Kan L, Dahmen U: Balancing innate immunity and inflammatory state via modulation of neutrophil function: A novel strategy to fight sepsis. J Immunol Res 2015;2015:187048.

44 Feinberg MW, Cao Z, Wara AK, Lebedeva MA, Senbanerjee S, Jain MK: Kruppel-like factor 4 is a mediator of proinflammatory signaling in macrophages. J Biol Chem 2005;280:38247-38258.

45 Tetreault MP, Wang ML, Yang Y, Travis J, Yu QC, Klein-Szanto AJ, Katz JP: Klf4 overexpression activates epithelial cytokines and inflammation-mediated esophageal squamous cell cancer in mice. Gastroenterology 2010;139:2124-2134.

46 Shen B, Smith RJ, Hsu YT, Chao L, Chao J: Kruppel-like factor 4 is a novel mediator of Kallistatin in inhibiting endothelial inflammation via increased endothelial nitric-oxide synthase expression. J Biol Chem 2009;284:35471-35478.

47 Liu F, Huang L: Improving plasmid DNA-mediated liver gene transfer by prolonging its retention in the hepatic vasculature. J Gene Med 2001;3:569-576.

48 Hu D, Gur M, Zhou Z, Gamper A, Hung MC, Fujita N, Lan L, Bahar I, Wan Y: Interplay between arginine methylation and ubiquitylation regulates KLF4-mediated genome stability and carcinogenesis. Nat Commun 2015;6:8419.

-49 Hao Z, Sheng Y, Duncan GS, Li WY, Dominguez C, Sylvester J, Su YW, Lin GH, Snow BE, Brenner D, You-Ten A, Haight J, Inoue S, Wakeham A, Elford A, Hamilton S, Liang Y, Zuniga-Pflucker JC, He HH, Ohashi PS, Mak TW: K48-linked KLF4 ubiquitination by E3 ligase Mule controls T-cell proliferation and cell cycle progression. Nat Commun 2017;8:14003.

50 Wang K, Zhou W, Cai Q, Cheng J, Cai R, Xing R: SUMOylation of KLF4 promotes IL-4 induced macrophage M2 polarization. Cell Cycle 2017;16:374-381. 